\title{
A routing Algorithm of Data Center Network Routing Energy- Saving Mechanism based on QoS
}

\author{
Shi Dong ${ }^{1,2,3^{*}}$ Xingang Zhang ${ }^{4 *} \mathrm{Ya} \mathrm{Li}^{1}$ \\ ${ }^{1}$ School of Computer Science and Technology Zhoukou Normal University, \\ Zhoukou 466000, China \\ ${ }^{2}$ School of Computer Science and Technology HuaZhong Universtiy of Science \\ and Technology, Wuhan 430079, China \\ ${ }^{3}$ Department of Computer Science and Engineering, Washington University in \\ St.Louis, Missouri 63130, USA \\ 4 School of Computer and Information Technology,Nanyang Normal University, \\ Nanyang 473061, China
}

Shi Dong and Xingang Zhang are corresponding author, njbsok@gmail.com*

\begin{abstract}
In order to meet the demand for network bandwidth and the reliability of the cloud computing applications, in recent years, many new data center network architectures have been put forward. They use the rich resources of network interconnection server to provide high performance and high fault-tolerant routing forwarding service. However, these "rich connection" architectures have network problems of energy consumption and energy use such as inefficient, resulted in the network of high operating costs and a large number of energy waste. In this paper, a typical energy efficiency of data center network architecture is studied from the perspective of network routing and scheduling. Different network flow scheduling and routing policy influence on energy consumption of data center network, in order to solve the above-mentioned problems, we put forward a kind of routing energy-saving mechanism network based on QoS which can improve the link utilization and the use of the network energy efficiency. The mechanism can effectively save the network energy consumption, and help us improve the average completion time of network flow.
\end{abstract}

Keywords: Data center network; Energy consumption model; Network energy efficiency; Energy efficient routing; QoS

\section{Introduction}

With the advent of the era of cloud computing, many large IT companies such as Microsoft, Google and amazon have built large-scale data center to provide users with a variety of cloud based application service, distributed computing and storage services, such as: Windows Azure, App Engine[1] [2], EC2[3] and GFS[4], etc. According to statistics, total energy consumption of data centers has increased from 61 billion KWH to 100 billion KWH from 2006 to 2011 in the United States [5].In 2011, around the world, global data center power consumption occupies $1.1 \sim 1.5 \%$ of the total global power consumption[6], and the percentage will increase to $8 \%$ by 2020 [7]. Energy consumption of data center mainly comes from the network, server, and the cooling energy consumption. Where network accounts for about $20 \%$ of the total energy consumption of data center $[8,9]$. Statistics show that the data center system utilization is $15 \%$ of the network energy consumption which will account for about 50\% of the total energy consumption of data center after deployment of efficient server equipment [9]. In recent years, many of the new data center network architectures [10-19], such as: Fat-Tree[11], 
BCube[12], DCell[14], etc., use abundant network equipment and routing forwarding service link in order to obtain a better network performance and reliability. However, these "connected" network topology at low load of energy efficiency is very low. This is because the data center network load is far lower than the peak load in most of the time. Thus can make that data center network has many free or the low utilization ratio of network equipment and link. Due to the low influence of the load change on current data center network equipment and components of energy consumption which is low compared with the energy consumption in full load [13], thus free and low utilization of network resources will waste a lot of invalid electricity. In view of the current typical "connection" data center network architecture [11-15] and in-depth analysis of their network energy efficiency features, we propose energy saving mechanism for data center network from the perspective of network routing and scheduling. The network energy efficiency analysis results presented in this paper can provide important reference and basis for architecture design and update of data center network. Our proposed energy flow routing and scheduling mechanism can effectively save the energy consumption of the data center network. The research results show that the new mechanism can reduce the energy consumption and operating costs of data center network. It can improve energy efficiency and reduce its carbon emissions.

The paper is structured as follows: Section 2 give out the general model of energy consumption and energy saving routing model; Section 3 discusses and analyzes several typical data center. Section 4 presents MERQ mechanism and experimental results in different scenes. Finally, section 5 concludes the paper and pointed out that the focus of future work.

\section{Data Center Network Energy Saving Routing Model}

Based on above-mentioned, the current user obtains energy consumption of network equipment only by observing the given maximum rated power of equipment in the specifications. However, the energy consumption values only points out the maximum power of network equipment and unable to accurately assess the energy consumption of the equipment in the actual runtime.

\subsection{General Model of Energy Consumption}

Existing studies have shown that the current energy consumption modular of network equipment mainly depends on the equipment configuration and rate of network traffic. Network equipment configuration includes exchange backboard type, card type, card number and rate of the network interface configuration [18]. An energy consumption modular of network equipment can be divided into three parts $[19,20]$ as shown in formula (1). $E(C, T)$ is the total energy consumption of network equipment, which is suitable for input parameters, $C$ and $T$ are respectively network equipment configuration and its network flow rate. $F(C)$ indicates that the energy consumption of network equipment of the inherent overhead, containing energy consumption of equipment and energy consumption of the cooling system, such as calculation cost of energy consumption for forwarding lookup and store cost of energy consumption for TCAM and RAM, etc. Total energy consumption for all the network equipment card and energy consumption of the network interface do not include the line card which is only associated with the type and amount of line card. $H(C, T)$ denotes the total energy consumption of all the equipment in the network interface, the energy consumption values of the network interface configuration and the network flow rate of the interface which is calculated using the formula (2). 


$$
\begin{gathered}
E(C, T)=F(C)+H(C, T) \\
H(C, T)=\sum_{i \in I} N_{i} * Q_{i}
\end{gathered}
$$

Where I denotes interface capacity set. $N_{i}$ refers to number of interface when interface capacity is i. $Q_{i}$ represents the power of interface.

The current data center network equipment used in low load with full load power consumption is relatively close, the network load changes have smaller impact on energy consumption of high transmission capacity of the network interface [21]. So the energy consumption model of the network equipment is simplified to formula (3). The $P(C)$ represents the energy consumption of the network equipment, it only depends on the configuration of the device parameter $C . j$ refers to the network interface in the network collection equipment, $D_{j}(C)$ represents the energy consumption of the network interface $\mathrm{J}$. In the formula (3), the energy consumption of the network equipment line card was incorporated into the inherent overhead $F(C)$. In the formula (3), if the energy of the switch interface are ESI, the energy consumption model can be further simplified as formula (4), where $M$ indicates the number of switches in the network interface.

$$
\begin{aligned}
& E(C)=F(C)+\sum_{j \in I} D_{j}(C) \\
& E(C)=F(C)+E S I * M
\end{aligned}
$$

\subsection{Energy Saving Routing Model}

Energy saving routing is to resolve the problem of network flow in traffic matrix T,R and scheduling policy $\mathrm{S}$ by calculating the transmission path. The final goal is to get minimum the energy consumption of network power transmission as shown in formula (5). Formula parameters $\mathrm{R}$ and $\mathrm{S}$ are pointed out in two dimensions in space and time on how to dispatch network flow, the parameter $\mathrm{R}$ denotes how to select the transmission path, parameter S points out when sending or suspend network flow according to the scheduling time for network flows. Formula (5) assumes that the inherent energy consumption per switch are $\mathrm{F}$ (including card energy consumption), energy consumption of single network interface are ESI. L (.) and $M$ (.) respectively refers to the number of switches working mode and the number of network interface which include the network routing $\mathrm{R}$ and flow scheduling policy $\mathrm{S}$.

$$
\begin{gathered}
\operatorname{Min} \operatorname{Energy}(T, R, S)=\operatorname{Min}(F * L(T, R, S)+E S I * M(T, R, S)) \\
\operatorname{QoS}_{i}(T, R, S) \geq(\leq) Q o S p_{i}, i=1,2,3, \ldots
\end{gathered}
$$

Because layer 3 switches interconnected servers with routing forwarding functions is usually used in the current data center network, these switches contains a large number of network interface which can provide highly reliable network interconnection and the high performance routing forwarding. Therefore, in this paper, the layer 3 switches with routing functions unity "switches" for short. According to the actual needs of different applications, the energy saving routing need to satisfy the multiple network service quality constraints, as shown in formula (6). Table 1 lists service quality constraints of several common network. 
Table.1 Common Network Service Quality Constraints

\begin{tabular}{cc}
\hline Constraint object & The commonly used constraints \\
\hline Network connectivity (choice) & $U_{i, j}=1, \forall i, j \in V$ \\
The network throughput (optional) & $\sum_{i \in T} Y_{i} \geq Q o S p_{1}$ \\
Network reliability (optional) & $\sum_{i \in T} Z_{i} \geq Q o S p_{2}$ \\
Network completion time (optional) & $\sum_{i \in T} W_{i} /|T| \leq Q o S p_{3}$ \\
\hline
\end{tabular}

From the above energy saving routing model shows the main influencing factors for energy consumption of data center network include routing policy $\mathrm{R}$ and flow scheduling strategy $\mathrm{S}$ in the network flow $\mathrm{T}$.

\section{Analysis on Energy Efficiency of Data Center}

In this paper, several typical data center and general architecture of the network energy consumption are analyzed. We use two indicators to compare and evaluate the energy efficiency of data center network architecture: one is the network energy consumption changing with load is proportional to the indicators, the second, and the network energy efficiency index. Energy efficiency is of great significance to research data center network, it can reflect the energy consumption characteristics of different data center architecture itself, the use effect of energy saving mechanism and balanced relationship between network performance and energy consumption.

Most current data center network does not support the dormancy technology for equipment, so this section researches on the total energy consumption of four kinds data center network architecture when there is no use any energy saving mechanism. The energy consumption only relies on the network topology characteristics, and used routing mechanism in the network and network traffic load.

Table 2. Four Kinds of Architecture in the Number of the Switch Interface and Server Interface

\begin{tabular}{lcr}
\hline $\begin{array}{l}\text { Data center network } \\
\text { architecture }\end{array}$ & $\begin{array}{c}\text { Number of 1Gbps switch } \\
\text { interface }\end{array}$ & $\begin{array}{r}\text { The server NIC interface } \\
\text { number }\end{array}$ \\
\hline Fat-Tree & $5 \mathrm{~N}$ & $\mathrm{~N}$ \\
BCube $(\mathrm{k}=2)$ & $3 \mathrm{~N}$ & $3 \mathrm{~N}$ \\
DCell $(\mathrm{k}=1)$ & $\mathrm{N}$ & $2 \mathrm{~N}$ \\
FiConn $(\mathrm{k}=1)$ & $\mathrm{N}$ & $3 \mathrm{~N} / 2$ \\
\hline
\end{tabular}

Given four kinds of architecture and the number of switch interface server NIC interface number in Table 2, you can find them with the server linear relationship between the number $\mathrm{N}$. When the network consists of $\mathrm{N}$ servers, because DCell and FiConn architecture are with only a single switch interface connection for each server, they contain the number of switch interface are all N. In the BCube, each server respectively keep connection in three different levels of switch, it contains $3 n$ exchange interface. The Fat-Tree containing $n$ pod-Tree architecture and server number $N=n^{3} / 4$. The number of the core layer, aggregation and edge switches are respectively $(\mathrm{n} / 2)^{2}, \mathrm{n}^{2} / 2$, so the number of total switch interface is $\left((n / 2)^{2}+n^{2} / 2+n^{2} / 2\right) * n=5 n^{3} / 4=5 N$. In the middle of the BCube topology, switch interface, aggregation switch interface and the top switch interface connects to the server using 1GBPS Ethernet interface. According to characteristics of BCube topology, assuming that each intermediate switches and switch of aggregation contains n network interfaces, on top of switch comprises two connectors and 30 interface 
connected to the server, the intermediate switches, aggregation and frame top switch respectively, the number of $n / 2, n, n^{2} / 4$, the total number of $N=5 n^{2}$ server. It can be found that Fat-Tree and BCube exchange interface architecture contains the largest number, because they are using three layer tree topology interconnection server. On the switch as the center, in the architecture of Fat-Tree and BCUBE because the server does not participate in the network routing and forwarding, each server using only one NIC interface connected to the switch, so N is a total number of NIC interface. In the BCube architecture, each server using three layer NIC interface are with different switches, so a total number of NIC interface is $3 \mathrm{~N}$. In DCell structure, each server using two interfaces connected in the same DCell0 switch and the adjacent DCell0 in the server, so a total of NIC interface is $2 \mathrm{~N}$. In the FiConn architecture, each server has two NIC interface, the other servers have a NIC interface, and therefore the total of NIC interface is $3 \mathrm{~N} / 2$. Accordingly, the server as the center of architecture contains more than switches NIC interface.

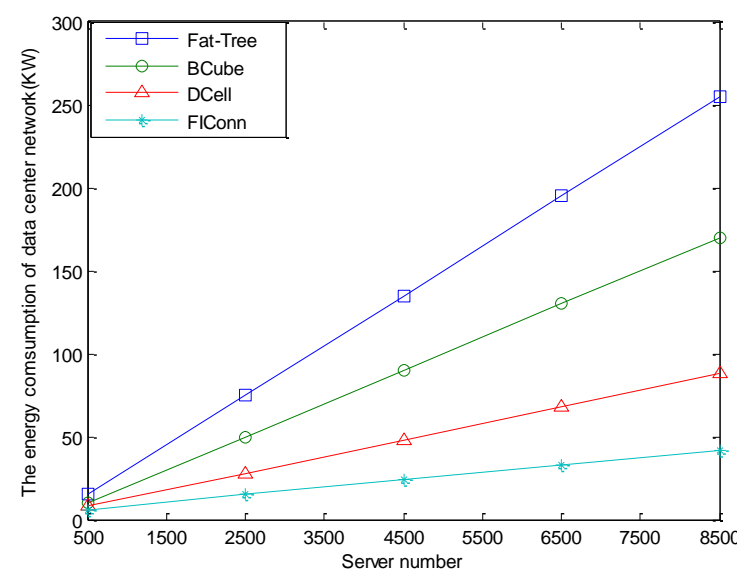

\section{Figure 1. The Energy Consumption Data Center in Different Number of Servers}

In Table 2, the interface number of NIC, switch and server can be calculated for each system structure. Figure 1 shows the energy consumption of four kinds of network architecture with the change of topology size. It can be seen from the diagram, the energy consumption of network structure linearly increases with the increase of the number of the server. At the same time, Fat-Tree and BCube have maximum total energy consumption of the network, because they as the center of architecture contains more switch interface, and the energy consumption of current switch interface is higher than the NIC server interface. DCell and FiConn two topology architecture have minimum network energy consumption. Because they use a recursive way to make switch interconnection in only 0 layer server and topology contains the least number of switch interface. By analysis on the total energy consumption ratio, we know energy consumption of data center network is mainly composed of energy consumption of switches NIC and the server.

\section{MERQ (Minimum Energy Route algorithm based on QoS) mechanism}

The basic idea of the mechanism is to use the way to find out the minimums energy cost $\mathrm{E}$ and path, which can meet the requirement of QoS for each node in the transmission to the exclusive network link bandwidth, and choose to reuse as much as possible for the network flow switch transmission path, thus effectively improve the utilization rate of switches and network link. The basic ideas of the algorithm are as follows: according to 
the size of the network topologic, make each network flow during transmission link bandwidth to monopolize the path. And exclusive for network bandwidth of network flow to choose energy-saving transmission path, allowing them to share in the network switch, in order to improve the exchange of energy efficiency. This problem can be described by an integer programming problem. It is be constructed by the Eq. (7).

$\operatorname{Min} \operatorname{Energy}(T, R, S)=\operatorname{Min}(F * L(T, R, S)+E S I * M(T, R, S))$

s.t.

$\operatorname{QoS}_{i}(T, R, S) \geq(\leq) Q o S p_{i} i=1,2,3, \ldots$

\begin{abstract}
Algorithm 1: MERQ algorithm
Input: G: data center network topology, Graph $\mathrm{G}=(\mathrm{V}, \mathrm{E})$

$q(i)$ : set QoS of the network flow

F: get a new network flow or a finished send network flow, including network flow information such as the size of the source and destination servers and flow
\end{abstract}

Output: QoS node, transmission path

1 if a collection network flow f completed then

$2 \quad \mathrm{M} \leftarrow|\mathrm{V}|$

3 for $\mathrm{k} \leftarrow 1$ to $\mathrm{M}$

4 for $\mathrm{i} \leftarrow 1$ to $\mathrm{M}$

$5 \quad$ for $\mathrm{j} \leftarrow 1$ to $\mathrm{M}$

$\mathbf{6} \quad \mathrm{d}_{\mathrm{ij}}^{(\mathrm{k})} \leftarrow \min \left\{\mathrm{d}_{\mathrm{ij}}{ }^{(\mathrm{k}-1)}, \mathrm{d}_{\mathrm{ik}}{ }^{(\mathrm{k}-1)}+\mathrm{d}_{\mathrm{kj}}{ }^{(\mathrm{k}-1)}\right.$;

$8\}$

9 Qnode $\leftarrow \Phi$;

10 for each $v i \in \mathrm{V}$

11\{

12 if Qnode $\leftarrow$ vi

13 Qnode $\leftarrow$ Qnode $+\{$ vi $\}$;

$14\}$

15 else

16 Dnode $\leftarrow\{$ vi $\}$;

17 return $\{<$ Qnode, epath $>$, e $\in$ QoS $\}$;

Minimum Energy Route algorithm based on QoS is shown in Algorithm1.

\title{
4.1 MERQ Function Module
}

MERQ mechanism consists of four function modules: network flow management module, the module precedence, scheduling and routing calculation module and network configuration module, the relationship between the modules as shown in figure 2 . 


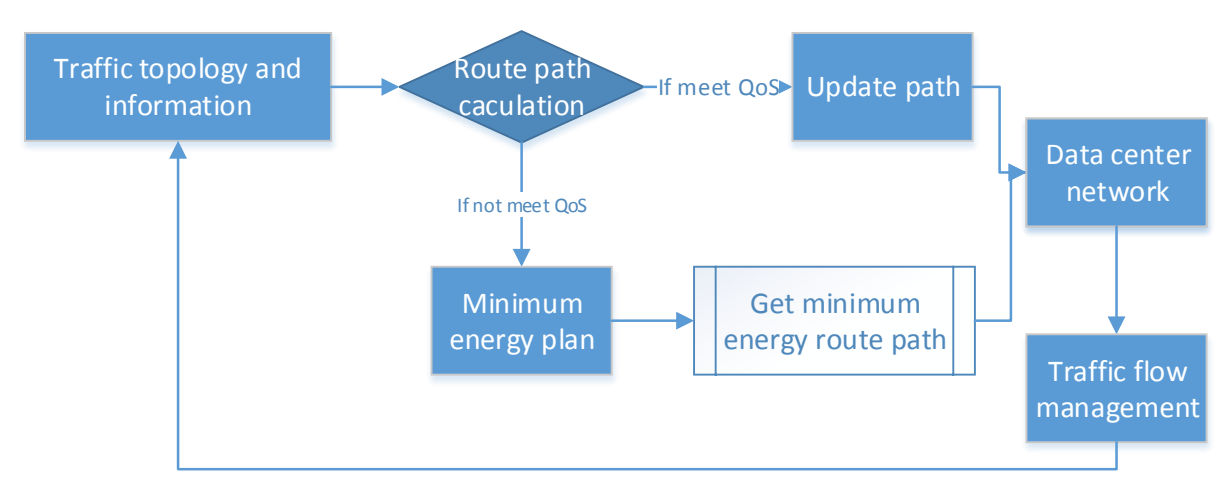

Figure 2. MERQ Function Module Diagram

Network flow management module is responsible for the collection and maintenance of the network topology information and traffic information. Information collection work can be done by existing network protocols and technologies, such as: simple network management protocol SNMP, and so on. This module is also responsible for maintaining and two network flow collection. Priority to compare module is used to compare different flow in a network of priority level. Network flow management module to use it for network flow sort. Scheduling and routing calculation module use it to determine whether a network flow exists path. In MERQ mechanism, using the network data flow amount as priority evaluation index, the smaller the network flow has the higher priority. Scheduling and routing calculation module is responsible for computing network flow state of scheduling and transmission path. The network flow management module collect the current topology information and network flow information of the new arrival. Network configuration module is responsible for the distributed network routing strategy to switch, server and flow scheduling strategy in the data center. When the network configuration module after receiving the output of routing calculation module, according to the results of routing strategy turn to exchange routing configuration, and scheduling policy announces network flow will flow to the appropriate sending server. The server decides when to send or suspend scheduling network flow according to the strategy.

\subsection{The Experimental Setup}

In the experiment, we use Fat-Tree topology with 30 pod and set up the network flow. Assumes that number of the network flow and time of arrival interval follows Poisson distribution. The total number of network flow our simulated is 14000. Each flow is scheduled to run immediately after arrival.

\subsection{The Experimental Results}

\subsubsection{Network Flow Arrival Interval}

Figure 3(a) shows energy consumption for the transmission flow network data in three strategies. It can be seen from the diagram, and compared two kinds of flow scheduling strategy based on ECMP routing, MERQ mechanism can effectively save the network energy consumption. For example, when the network flow average arrived at intervals of $3 \mathrm{~ms}$, using MERQ mechanism of network energy consumption was only about $60 \%$ of the energy consumption when using BSECMP strategy. In addition, with the increase of network flow mean arrival interval ( $\mathrm{ms}$ increases from 0.2 to $8 \mathrm{~ms}$ ), using three strategies of network energy consumption will be increased. This is because the smaller is the average network flow arrival interval, the greater is the number of flows in the network, at the same time, thus 
will make the network flow have more opportunity to fully share the switches for transmission, enhancing the using rate of network equipment and network energy use efficiency.

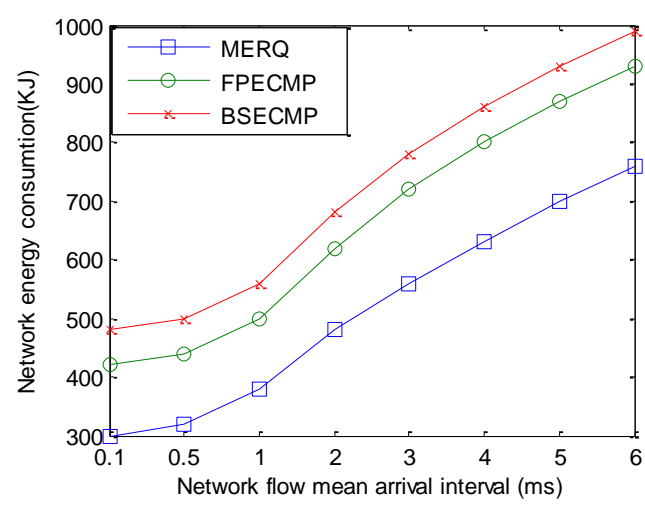

(a) network energy consumption

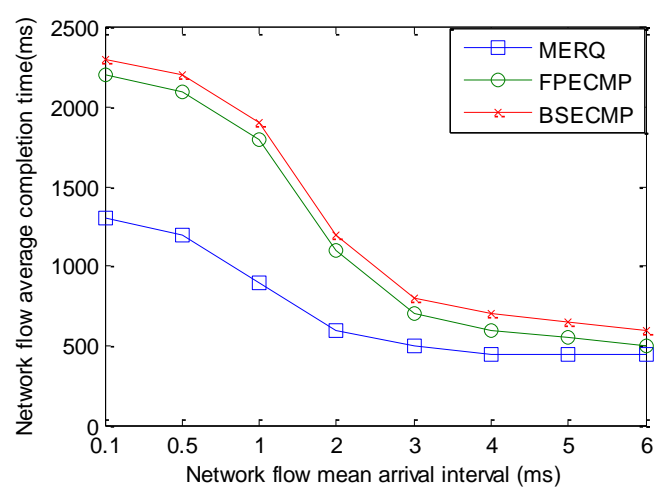

(b) average completion time

Figure 3. Using three Different Transmission Network Flow Mean Arrival Interval

Figure 3(b) shows average completion time in different flow arrival interval using three strategies. Experimental results show that MERQ mechanism can get better network flow average completion time compared with BSECMP and FPECMP strategy under the different network flow arrival interval, especially when the network flow arrival interval is small, its advantage is more obvious. For example, when the average arrived at intervals of $0.2 \mathrm{~ms}$, MERQ mechanism can reduce more than $40 \%$ of the average completion time network flow compared with BSECMP strategy and strategy of FPECMP. In addition, from figure 3(b) we can find the average completion time decreases with the increasing of arrival interval. This is because the network flow mean arrival interval is the greater, the transmission probability of competing will is smaller, and so faster transmission will be completed in the network.

\subsubsection{Network Flow Size Effect}

Figure 4(a) shows the network energy consumption in different size of network flow using three kinds of scheduling mechanism. The abscissa in the Figure 4(a) indicates average size of network flow obeying exponential distribution. Network energy consumption of three flow scheduling strategy increase as the increase of size of network flow which presents linear growth. This is because the network flow is the greater, data transmission in time is longer, so the flow switches used as transfer consume the higher energy. Under the different size of the network flow, MERQ mechanism has better performance on energy efficiency than BSECMP strategy and FPECMP strategy. For example, when the average size of network flow is 128 $\mathrm{MB}$, network energy consumption using MERQ mechanism only is $770 \mathrm{KJ}$, while network energy consumption of other two kinds of scheduling strategy based on ECMP are higher than $1000 \mathrm{KJ}$. The three direction curves in Figure 4(a) keeping the relative parallel indicate that the network flow under the three strategies have energy gap in different size of the network. For all kinds of network flow size (32 MB to $160 \mathrm{MB}$ ), network energy consumption using MERQ mechanism accounts for about $65 \%$ of the energy consumption of using BSECMP strategy.

Figure 4(b) shows the average completion time flow under different size of network flow using three kinds of scheduling policy. The experimental results show that average completion time of the three strategies increases as the size of the network flow, because the network flow is the greater, transfer time used to deal with the amount of data will be longer. Under the different size of the network flow, MERQ mechanism can effectively improve the 
average completion time of network flow, and the network flow is the greater, improvement effect is the more obvious. For example, when the network flow average size is $96 \mathrm{MB}$, average complete time using MERQ mechanism is 1.1 seconds, while using the other two flow scheduling strategy based on ECMP, the average completion time will reach more than 2 seconds.

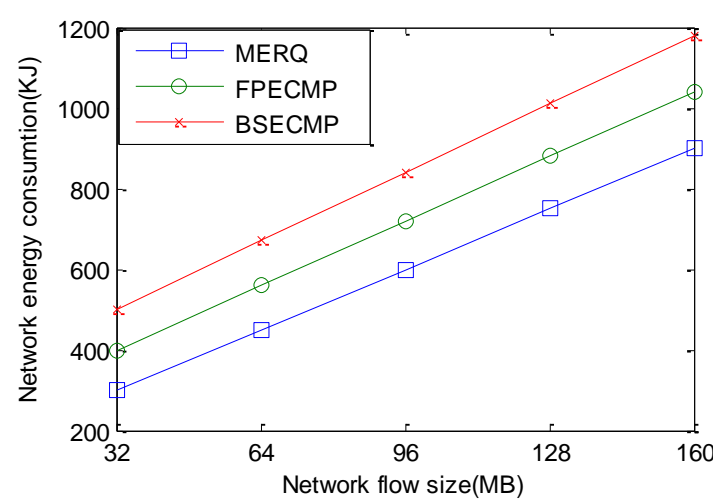

(a) network energy consumption

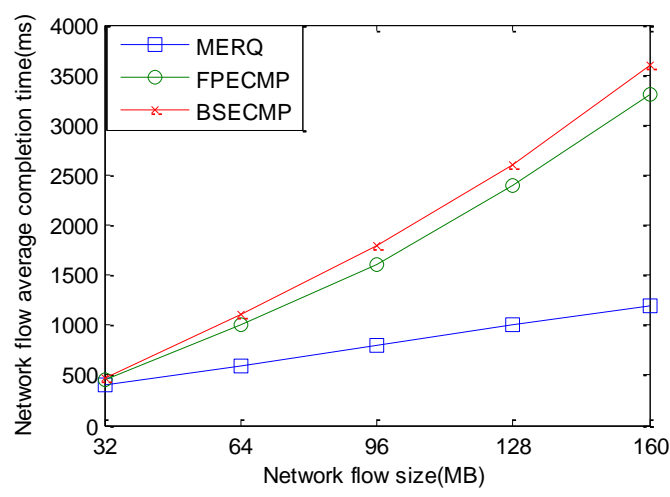

(b) average completion time

\section{Figure 4. The Use of Three Kinds of Flow under Different Size of Network Flow Scheduling Strategy}

\subsubsection{Network Flow Quantity}

Figure 5(a) shows the network energy consumption in different network flow number using the three kinds of flow scheduling policy. The experimental results show that network energy consumption of the three strategies increases with the increase of the number of the data flow, because in the network topology size scale, the flow rate and flow phase at the same time, transmitting the data flow of the required number of network energy consumption. Compared with two kinds of flow scheduling strategy based on ECMP, use MERQ mechanisms needed to transmit the same number of network flow network minimum energy consumption. For example, network energy consumption of MERQ mechanism using 20000 transmission flow required is about $930 \mathrm{KJ}$, while energy consumption of using BSECMP and FPECMP strategy transmitting the same number of network flows respectively need $1450 \mathrm{KJ}$ and $1250 \mathrm{KJ}$.

Figure 5(b) shows the flow average completion time in different network flow number using the three kinds of scheduling policy. The experimental results show that in different number of network transmission flow, using MERQ mechanism can effectively reduce the average completion time of network flow. When the network flow number is 5000, for example, flow average completion time using MERQ mechanism is only $630 \mathrm{~ms}$, while flow average completion time using BSECMP and FPECMP strategy are $1030 \mathrm{~ms}$ and $930 \mathrm{~ms}$ respectively. In addition, as can be found in Figure 5(b), three wires are close to the horizontal direction which indicates the change of flow average completion time in different network flow quantity using three scheduling policies. 


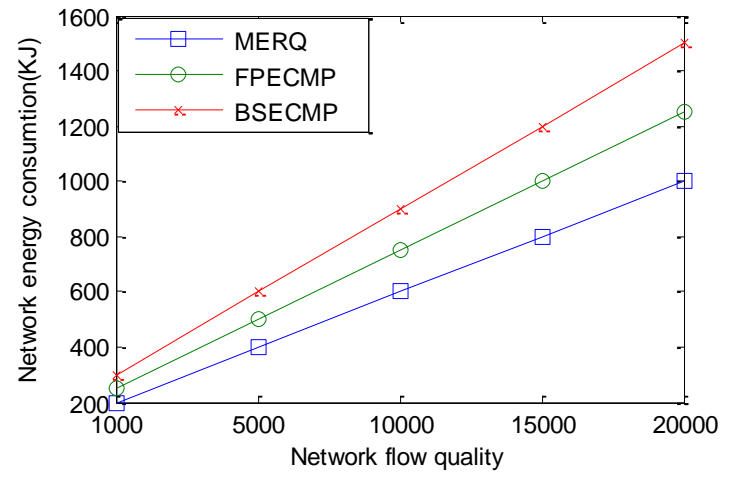

(a) network energy consumption

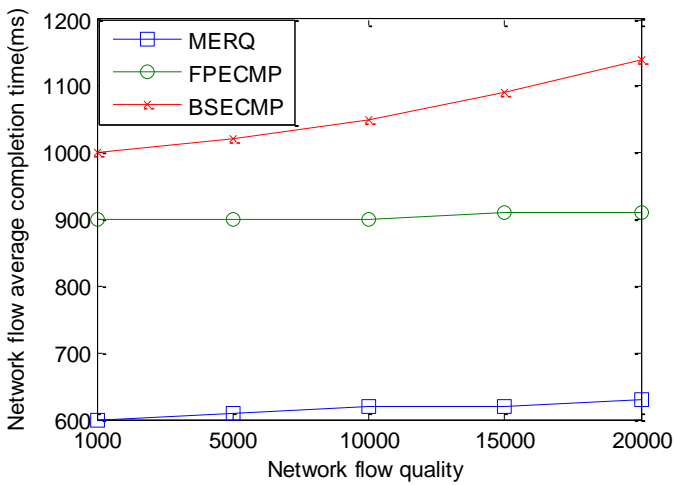

(b) average completion time

\section{Figure 5. Using Three Different Flow Scheduling Policy Transmission Network Flow Quantity}

\subsubsection{QoS}

In Figure 6(a), QoS value is given, we can see, when QoS=50, energy consumption of MERQ is $1200 \mathrm{KJ}$. While FPECMP and BSECMP can reach $1400 \mathrm{KJ}$ and $1750 \mathrm{KJ}$, and the performance of all algorithms shows decreasing as QoS increasing. It is because, with the QoS scaled up, the condition requirement is easier met, thus network energy consumption is smaller. While the FPECMP and BSECMP both can not consider the QoS, so this will affect the experiment result.

Figure 6(b) shows the difference of average completion time in three methods (FPECMP, BSECMP, and MERQ), when QoS=250, the average completion time is lowest in the MERQ, which is just $500 \mathrm{~ms}$.It is because, with the QoS increasing, the computing time is shorten, so the average completion time is more short as QoS increasing.

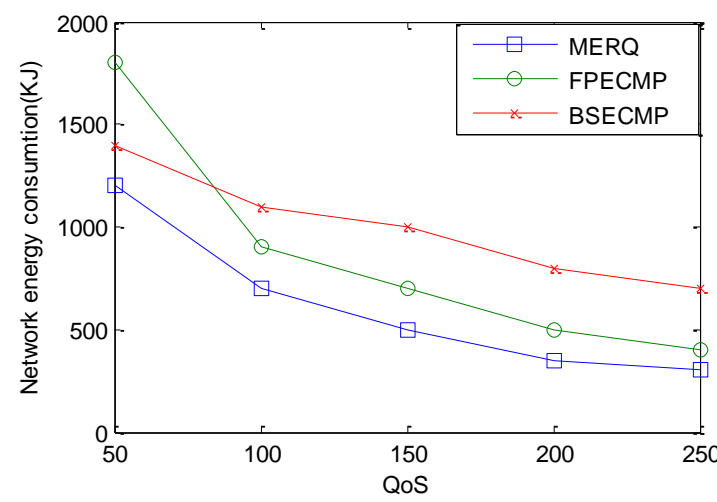

(a) network energy consumption

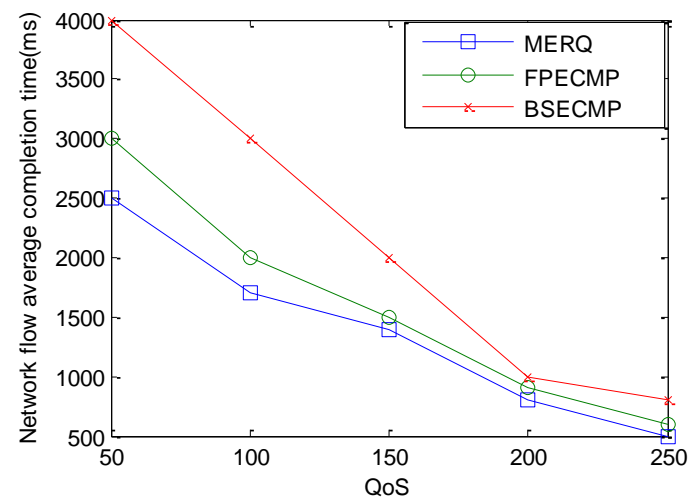

(b) average completion time

Figure 6. Using Three Different Transmission Network Flow QoS

\subsubsection{Flow Distribution Network}

Figure 7(a) shows network energy consumption under different network flow distribution using three flow scheduling strategy. The experimental results show that the MERQ mechanism can effectively reduce the network energy consumption used to transmit data flow, especially when the server using percentage is small, energy-saving effect is more obvious. For example, when the server using percentage is 10\%, network energy consumption using MERQ mechanism is only about 35\% of the energy consumption using BSECMP strategy. In addition, when the server using percentage reduces to less than $30 \%$, network 
energy consumption using BSECMP strategy will rise sharply, because BSECMP strategy transfer relatively concentrate distribution network flows intensify network link bandwidth competition, lead to significantly prolong switches working time. It can be seen from the diagram, network energy consumption of three flow scheduling strategy reduce with the increase of server used percentage. This is because when the scale of network topology and network flow arrival interval phase at the same time, the server using percentage is higher, the network flow will be more evenly distributed on the server, when the network flow transmitted can share more fully switch, so as to improve the utilization of switches and network energy use efficiency.

Figure 7(b) shows the average completion time in different network flow distribution using three scheduling policies. It can be seen from the diagram, as the growth of the server using percentage of network, flow average completion time will be reduced. Because the network flow distribution is average, the probability of each transmission competition is the smaller, so that they can more quickly complete the transfer. In addition under the different network flow distribution, MERQ mechanism can effectively improve the average completion time of network flow.

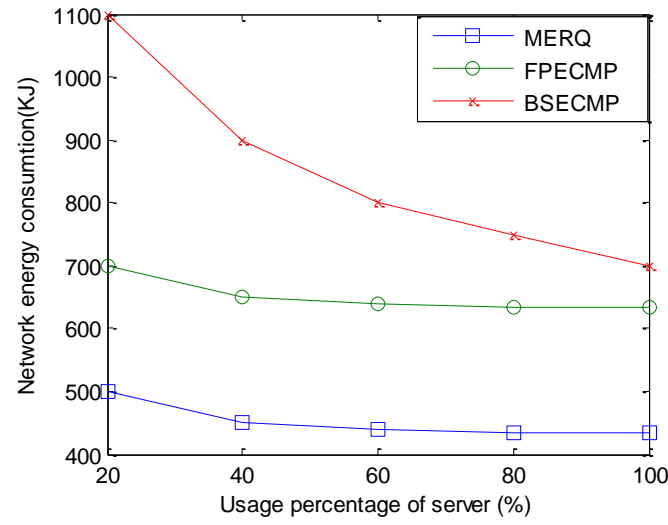

(a) network energy consumption

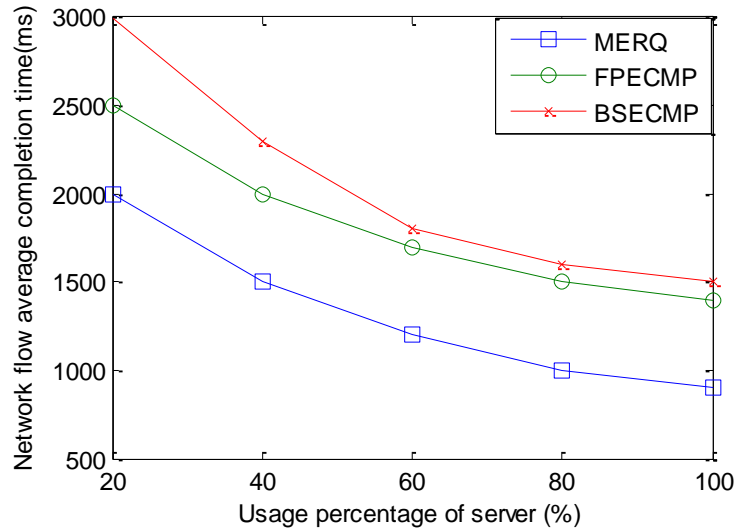

(b) average completion time

\section{Figure 7. The use of Three Kinds of Flow under Different Network Flow Distribution}

We study how to jointly use of network routing and flow scheduling method to realize the data center network energy savings. First we give out energy flow network routing and scheduling problem description, and through analysis of network flow scheduling sequence and the influence of the route choice on network energy consumption. Network flow scheduling and routing energy saving mechanism, called MERQ mechanism is proposed. The basic idea is to preempt the way according to the volume of flow priority scheduling network flow, make its can monopolize in the process of transmission network link bandwidth, so as to improve the use of network link utilization and energy efficiency. The experimental results show that MERQ mechanism can effectively save the energy consumption of the data center network compared with the two kinds of network flow scheduling strategy based on ECMP routing, at the same time, it also can improve the average completion time of the network flow.

\section{Conclusion}

This paper proposes the energy saving mechanism (MERQ) combining flow scheduling and routing, it makes the network flow transmitted in the form of an exclusive network link bandwidth, can effectively improve the utilization rate of network link and the network 
energy use efficiency. In this article, we research MERQ mechanism in different scale of network flow arrival interval, network topology. The experimental results show that MERQ mechanism can effectively reduce the energy consumption data center network and improve the average completion time of the network flow compared with the two kinds of network flow scheduling strategy based on ECMP routing.

\section{Acknowledgements}

The authors would like to thank the anonymous reviewers for helpful comments which helped them improve the technical quality of the paper. This paper is supported by Project supported by the National Natural Science Foundation of China (Grant No.U1504602), China Postdoctoral Science Foundation (Grant No.2015M572141), Science and Technology Plan Projects of Henan Province (Grant No.162102310147), Henan Science and Technology Department of Basic and advanced technology research projects (132300410276, 142300410339), Education Department of Henan Province Science and Technology Key Project Funding (Grant No.14A520065).

\section{References}

[1] Windows Azure [EB/OL], [2013-3-15], http://www.microsoft.com/windowsazure/, (2013).

[2] Google App Engine [EB/OL], [2013-3-15], https://developers.google.com/ appengine/, (2013).

[3] Amazon Elastic Compute Cloud [EB/OL], [2013-3-15], http://aws.amazon.com/ec2/, (2013).

[4] S. Ghemawat, H. Gobioff and S. Leung, "The Google File System", Proc. of the 19th ACM symposium on Operating systems principles (SOSP), (2003)

[5]U. S. Environmental Protection Agency, Data Cente Report to Congress [EB/OL], (2013).

[6]J. G. Koomey, "Growth in dat center electricity use 2005 to 2010”, Analytics Press, (2011).

[7] P. X. Gao, A. R. Curtis, B. Wong and S. Keshav, "It's Not Easy Being Green”, Proc. of the ACM SIGCOMM, (2012).

[8] A. Greenberg, J. Hamilton, D. A. Maltz and P. Patel, "The cost of a cloud: research problems in data center networks", ACM SIGCOMM Computer Communication Review, vol. 39, no. 1, (2009), pp. 68-73.

[9] D. Abts, M. R. Marty, P. M. Wells, P. Klausler and H. Liu, "Energy proportional datacenter networks", Proc. of the 37th annual international symposium on Computer architecture (ISCA), (2010).

[10]Cisco Systems, Cisc Data Center Infrastructure 2.5 Design Guide [EB/OL], (2013).

[11]M. Al-Fares, A. Loukissas and A. Vahdat, "A scalable, commodity data center network architecture", Proc. of the ACM SIGCOMM, (2008).

[12]C. Guo, G. Lu and D. Li, "BCube: a high performance, server-centric network architecture for modular data centers", Proc. of the ACM SIGCOMM, (2009).

[13] A. Greenberg, J. R. Hamilton and N. Jain, "VL2: a scalable and flexible data center network", Proc. of the ACM SIGCOMM, (2009).

[14] C. Guo, H. Wu, K. Tan, L. Shi, Y. Zhang and S. Lu, "DCell: a scalable and fault-tolerant network structure for data centers", Proc. of the ACM SIGCOMM, (2008).

[15] D. Li, C. Guo, H. Wu, K. Tan, Y. Zhang and S. Lu, "FiConn: Using Backup Port for Server Interconnection in Data Centers", Proc. of the IEEE INFOCOM, (2009).

[16] Chen K, Singla A and Singh A, "OSA: an optical switching architecture for data center networks with unprecedented flexibility", IEEE/ACM Transactions on Networking, vol. 22, no. 2, (2014), pp. 498-511.

[17] Wang T, Su Z and Xia Y, "Towards cost-effective and low latency data center network architecture", Computer Communications, vol. 82, (2016), pp. 1-12.

[18] R. N. Mysore, A. Pamboris, N. Farrington and N. Huang, "PortLand: A Scalable Fault-Tolerant Layer 2 Data Center Network Fabric", Proc. of the ACM SIGCOMM, (2009).

[19] H. Abu-Libdeh, P. Costa, A. Rowstron, G. O'Shea and A. Donnelly, "Symbiotic routing in future data centers", Proc. of the ACM SIGCOMM, (2010).

[20] H. Wu, G. Lu, D. Li, C. Guo and Y. Zhang, "MDCube: A High Performance Network Structure for Modular Data Center Interconnection", Proc. of the ACM CoNext, (2009).

[21] D. Li, M. Xu, H. Zhao and X. Fu, "Building Mega Data Center from Heterogeneous Containers", Proc. of the 19th annual IEEE International Conference on Network Protocols (ICNP), (2011). 


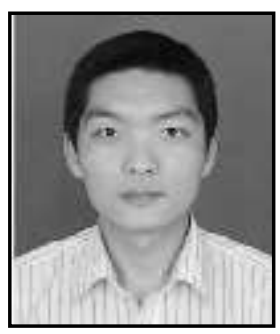

Shi Dong. Received the M.E. degree in computer application technology from University of Electronic Science and Technology of China in 2009 and the $\mathrm{PhD}$ in computer application technology from Southeast University in 2013. Currently, he is an associate in the School of Computer Science and Technology at Zhoukou Normal University and he also works as post doctor researcher in Huazhong University of Science and Technology. He is member of China Computer Federation. $\mathrm{He}$ is a visiting scholar in Washington University in St. Louis. His research interests include distributed computing, network management and evolutionary algorithm.

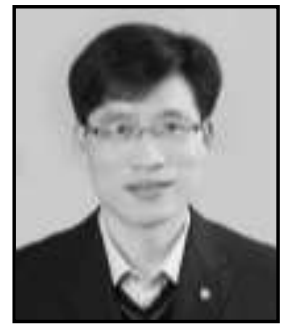

Xingang Zhang. Received the Master's degree in computer application technology from Huazhong University of Science and Technology in 2010.Currently, he is an associate professor in the School of Computer and Information Technology at Nanyang Normal University. He is senior member of China Computer Federation. His research interests include distributed computing and computer network.

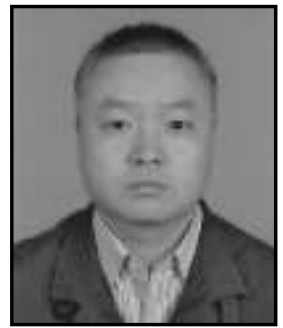

Ya Li. Received the B.Sc. degree in Computer Science and Technology from the Northeast Normal University and the M.Sc. degree in Computer application Technology from the Beijing Jiaotong University, China, in 1995 and 2005, respectively. He is currently doing research in the Computer Application Technology and Mobile Internet Technology. 
International Journal of Grid and Distributed Computing

Vol. 10, No. 1 (2017) 